\title{
Protective role of thymoquinone in sepsis-induced liver injury in BALB/c mice
}

\author{
FEI WANG ${ }^{1 *}$, XIONG LEI $^{2 *}$, YUE ZHAO $^{2}$, QINGGONG YU ${ }^{1}$, QIANWEI LI ${ }^{1}$, HUI ZHAO $^{3}$ and ZUOWEI PEI ${ }^{4}$ \\ ${ }^{1}$ Department of Gastroenterology, Affiliated Zhongshan Hospital of Dalian University, Dalian, Liaoning 116001; \\ ${ }^{2}$ Graduate School of Dalian Medical University, The First Clinical College, Dalian, Liaoning 116044; \\ Departments of ${ }^{3}$ Vascular Surgery and ${ }^{4}$ Cardiology, Affiliated Zhongshan Hospital of Dalian University, \\ Dalian, Liaoning 116001, P.R. China
}

Received September 16, 2018; Accepted June 6, 2019

DOI: $10.3892 /$ etm.2019.7779

\begin{abstract}
Sepsis increases the risk of developing liver injury. Previous studies have demonstrated that thymoquinone (TQ) exhibits hepatoprotective properties in vivo as well as in vitro. The present study aimed to investigate the underlying mechanisms of the protective effects of TQ against liver injury in septic BALB/c mice. Male BALB/c mice (age, 8 weeks) were randomly divided into four groups, namely, the control, TQ (50 mg/kg/day) treatment, cecal ligation and puncture (CLP), and TQ + CLP groups. CLP was performed following gavage of TQ for 2 weeks. At $48 \mathrm{~h}$ post-CLP, the histopathological alterations in the liver tissue (LT) and plasma levels of serum alanine aminotransferase (ALT), aspartate aminotransferase (AST) and alkaline phosphatase (ALP) were assessed. The present study evaluated microtubule-associated protein light chain 3 (LC3), sequestosome-1 (p62) and beclin 1 protein expression by western blotting and immunostaining, as well as interleukin (IL)-6, IL-1 $\beta$, IL-10, monocyte chemoattractant protein-1 (MCP-1) and tumor necrosis factor- $\alpha$ (TNF- $\alpha$ ) mRNA expression by RT-qPCR. The results of the present study indicated that administration of TQ to mice reduced the histological alterations caused by CLP in LT. TQ inhibited the plasma levels of ALT,
\end{abstract}

Correspondence to: Dr Zuowei Pei, Department of Cardiology, Affiliated Zhongshan Hospital of Dalian University, 6 Jiefang Street, Dalian, Liaoning 116001, P.R. China

E-mail: pzw_dl@163.com

*Contributed equally

Abbreviations: ALP, alkaline phosphatase; ALT, alanine aminotransferase; AST, aspartate aminotransferase; CLP, cecal ligation and puncture; IHC, immunohistochemical; IL, interleukin; LC3, microtubule-associated protein light chain 3; LT, liver tissue; MCP-1, monocyte chemoattractant protein-1; p62, sequestosome-1; TNF- $\alpha$, tumor necrosis factor- $\alpha$; TQ, thymoquinone; WBA, western blot analysis

Key words: sepsis, thymoquinone, liver injury, autophagy, BALB/c mice
AST and ALP in the CLP group. TQ significantly inhibited the elevation of p62, IL-1 $\beta$, IL-6, MCP-1 and TNF- $\alpha$ levels as well as increased the LC3, beclin 1 and IL-10 levels in LT. PI3K expression in the TQ + CLP group was significantly decreased compared with that in the CLP group. TQ treatment effectively modulated the expression levels of p62, LC3, beclin 1, PI3K and proinflammatory cytokines, and may be an important agent for the treatment of sepsis-induced liver injury.

\section{Introduction}

Sepsis is a major cause of mortality and critical illness in intensive care units and is defined as a life-threatening organ dysfunction caused by a dysregulated host response against infection that triggers systemic inflammatory response syndrome $(1,2)$. Among the organ failures involved in sepsis, hepatic dysfunction is a prominent complication that contributes to an increased risk of development of multiple organ failure and is a strong independent predictor of mortality $(3,4)$.

Clinically, the survival outcome of patients with sepsis remains unimproved although several clinical trials employ novel agents to modulate the hyperreactive inflammatory response, and a wide spectrum of effective antibiotics are available against sepsis $(5,6)$. Previous studies have demonstrated a close interaction between cecal ligation and puncture (CLP)-induced septic organ failure and cell dysfunction due to autophagy (7-9). Autophagy is a well-conserved intracellular lysosomal degradation process in which specific enzymes degrade the damaged or abnormal cellular components and recycle the redundant or inefficient components in cells $(3,10)$. Autophagy exhibits an inducible response towards stress, including tissue injury, oxidative stress, infection and protein aggregation (3). Several studies have indicated that autophagy is activated after performing CLP in animal models of sepsis $(7,11)$. Furthermore, multiple studies revealed that autophagy serves an important role in liver physiology and pathology following CLP $(12,13)$. Various clinical experiments have suggested that novel strategies that focus on cell survival, in which an important role is served by autophagy, may improve the survival outcome in patients with septic shock $(14,15)$. Therefore, further investigations are required to develop novel therapeutic interventions against sepsis. 
Several studies investigated herbal medicines with an increasing interest in pre-clinical and clinical studies (16-18). Multiple studies support the high efficiency of medicinal plants in the prevention and cure of a wide range of diseases $(16,19)$. Thymoquinone (2-isopropyl-5-methylbenzo-1,4-quinone; TQ), an agent extracted from the Nigella sativa (black cumin) plant, is used in Middle and Far Eastern countries as a traditional medicine to treat a wide range of pathological conditions (20). According to traditional medicine, TQ exhibits various types of therapeutic properties, including antioxidative, anti-inflammatory, immunomodulatory and antimicrobial activities (21). However, the effect of TQ on CLP-induced septic liver injury remains unclear. Therefore, in the present study, a murine model that simulates human peritonitis was established by performing CLP in order to understand the effect of TQ on CLP-induced septic liver injury.

\section{Materials and methods}

Maintenance of animal models. All animal experiments were approved by the Animal Studies Committee of the Affiliated Zhongshan Hospital of Dalian University. Male BALB/c mice (n=48; weight, 18.8 $\pm 1.3 \mathrm{~g}$; age, 8 weeks) were purchased from Beijing Vital River Lab Animal Technology Co., Ltd. All mice were housed under constant conditions, including $23-25^{\circ} \mathrm{C}$ temperature, 40-60\% humidity and a 12-h light/dark cycle with ad libitum access to food and water.

Development of a murine model of sepsis. To induce polymicrobial sepsis, a murine model was established by performing CLP according to a previously described method (22). The mice were anesthetized with sodium pentobarbital $(50 \mathrm{mg} / \mathrm{kg}$; intraperitoneal injection). After the peritoneum was surgically opened and the bowel was exposed, a section (two-thirds) of the cecum was tied and punctured once with a 21-gauge needle. A gentle pressure was applied at the perforation site to extrude a small amount of feces, which were placed in the peritoneal cavity. Finally, the laparotomy site was stitched. Sham-operated (control and TQ group) mice underwent the same procedure that involved surgical opening of the peritoneum and exposing the bowel; however, the ligation and needle perforation of cecum were not performed. Male BALB/c mice ( $n=48$; age, 8 weeks) were randomly divided into four groups ( $\mathrm{n}=12 /$ group), namely, the control, TQ (50 mg/kg/day; Sigma-Aldrich; Merck KGaA), CLP and TQ + CLP groups. In the TQ + CLP group, CLP was performed following gavage of TQ for 2 weeks. Mice exhibiting signs of humane endpoints were immediately sacrificed. Before the sacrifice of animals at $48 \mathrm{~h}$ post-CLP, the mortality rates of CLP group and TQ + CLP group were 16.67 and $8.33 \%$ respectively, and no mice died in the control and TQ groups. At $48 \mathrm{~h}$ post-CLP, all mice that survived were sacrificed, and blood samples were obtained from their inferior vena cava, collected in serum tubes and stored at $-80^{\circ} \mathrm{C}$ until further use. The coronal sections of liver tissues (LTs) were fixed in $10 \%$ formalin for $30 \mathrm{~min}$ at room temperature, dehydrated in $75 \%$ ethanol overnight then embedded in paraffin for histological evaluation. The remnants of LTs were snap-frozen $\left(-80^{\circ} \mathrm{C}\right)$ and used to perform mRNA or immunoblotting analyses. All animal experiments were performed according to the Guide for the Care and Use of Laboratory Animals (23). The present study was approved by the Animal Studies Committee of the Affiliated Zhongshan Hospital of Dalian University.

Serum analysis. The concentrations of alanine aminotransferase (ALT; cat. no. G0050), aspartate aminotransferase (AST; cat. no. G0073) and alkaline phosphatase (ALP; cat. no. G0040) in the serum were estimated using ELISA kits according to the manufacturer's protocols (Shanghai Westang Bio-Tech Co., Ltd).

Hematoxylin and eosin $(H \& E)$ staining. Sections (4- $\mu$ m thickness) were serially cut to perform the morphometric analysis of LTs. These sections were stained with hematoxylin for $15 \mathrm{~min}$ and eosin for $5 \mathrm{~min}$ at room temperature to perform histological analysis.

Morphological and IHC analyses. IHC analysis was performed using a histone simple stain kit (cat. no. 414341F; Nichirei Corporation) according to the manufacturer's protocol. Paraffin-embedded sections were deparaffinized using xylene and rehydrated in the descending ethanol series. These sections (4- $\mu \mathrm{m}$ thickness) were blocked with $3 \% \mathrm{H}_{2} \mathrm{O}_{2}$ in methanol for $15 \mathrm{~min}$ at room temperature to inactivate the endogenous peroxidases then incubated at room temperature for $1 \mathrm{~h}$ with primary antibodies against sequestosome-1 (cat. no. 18420-1-AP; p62; rabbit anti-p62 antibody; 1:200 dilution; Wuhan Sanying Biotechnology) and microtubule-associated protein light chain 3 (cat. no. 14600-1-AP; LC3; rabbit anti-LC3 antibody; 1:200 dilution; Wuhan Sanying Biotechnology). The secondary antibody from Histone Simple Stain kit (cat. no. 414341F; Nichirei Corporation) was placed on the tissue for $30 \mathrm{~min}$ at room temperature. Histological examination was performed under a light microscope (magnification, x400; Olympus BX43; Olympus Corporation).

RNA isolation and reverse transcription-quantitative PCR $(R T-q P C R)$. Total RNA was isolated from the LTs using ISOGEN reagent (Nippon Gene Co., Ltd.) according to the manufacturer's protocol. Complementary DNA (cDNA) was synthesized from total RNA using a first-strand cDNA synthesis kit (SuperScript VILO cDNA synthesis kit; Thermo Fisher Scientific, Inc.) according to the manufacturer's protocol. The specific gene expression levels were quantitatively analyzed by performing qPCR using fluorescent SYBR Green technology (Light Cycler; Roche Molecular Diagnostics). $\beta$-actin cDNA was amplified and quantified in each cDNA preparation to normalize the relative expression of target genes. The thermocycling conditions were as follows: $95^{\circ} \mathrm{C}$ for $3 \mathrm{~min} ; 95^{\circ} \mathrm{C}$ for $15 \mathrm{sec} ; 60^{\circ} \mathrm{C}$ for $15 \mathrm{sec} ; 72^{\circ} \mathrm{C}$ for $1 \mathrm{~min}(35$ cycles $)$; and $72^{\circ} \mathrm{C}$ for $10 \mathrm{~min}$. The relative expression of genes was determined using the $2^{-\Delta \Delta \mathrm{Cq}}$ method (24) with normalization to $36 \mathrm{~B} 4$ expression. The primer sequences used in the present study are listed in Table I.

Western blot analysis (WBA). Proteins were extracted from the LT using radioimmunoprecipitation assay buffer (cat. no. P0013B; Beyotime Institute of Biotechnology). Protein concentration was detected with a bicinchoninic acid Protein Assay kit (Beyotime Institute of Biotechnology). Protein samples $(20 \mu \mathrm{g}$ per lane) were separated by SDS-PAGE 
Table I. Primer oligonucleotide sequences.

\begin{tabular}{ll} 
Gene & \multicolumn{1}{c}{ Primer sequences $\left(5^{\prime}-3^{\prime}\right)$} \\
\hline TNF- $\alpha$ & F: TCTCATGCACCACCATCAAGGACT \\
& R: ACTTGCAGAACTCA \\
IL-6 & F: TACCAGTTGCCTTCTTGGGACTGA \\
& R: TAAGCCTCCGACTTGTGAAGTGGT \\
IL-1 $\beta$ & F: TGCCCCTTTTGACAGTGAT \\
& R: TGTGCTGCTGCGAGATTTGA \\
MCP-1 & F: ACTGAAGCCAGCTCTCTCTTCCTC \\
& R: TTCCTTCTTGGGTCAGCACAGAC \\
IL-10 & F: GACAACATACTGCTAACCGACT \\
& R: ATCACTCTTCACCTGCTCCAC \\
$\beta$-actin & F: CGATGCCCTGAGGGTCTTT \\
& R: TGGATGCCACAGGATTCCAT
\end{tabular}

F, forward; R, reverse; IL, interleukin; MCP-1, monocyte chemoattractant protein- 1 ; TNF- $\alpha$, tumor necrosis factor- $\alpha$.

(10\% gel), and proteins were transferred onto polyvinylidene difluoride (PVDF) membranes (Immobilon; EMD Millipore). PVDF membranes were blocked in TBS with $0.1 \%$ Tween-20 containing 5\% skimmed milk for $2 \mathrm{~h}$ at room temperature, incubated in primary antibody diluent (cat. no. P0023A; Beyotime Institute of Biotechnology) and gently agitated overnight at $4^{\circ} \mathrm{C}$. Primary antibodies against p62 (cat. no. 18420-1-AP; 1:1,000 dilution), LC3 (cat. no. 14600-1-AP; 1:1,000 dilution), beclin 1 (cat. no. 11306-1-AP; 1:1,000 dilution), PI3K (cat. no. 20584-1-AP; 1:1,000 dilution) all from Wuhan Sanying Biotechnology, and anti- $\beta$-actin (cat. no. 4970; 1:1,000 dilution; Cell Signaling Technology, Inc.) were utilized in the present study. Membranes were then further incubated with horseradish peroxidase-conjugated goat anti-rabbit Immunoglobulin G (cat. no. 7074; 1:1,000 dilution; Cell Signaling Technology, Inc.) for $1 \mathrm{~h}$ at room temperature. WBA was performed as three independent experiments. The protein levels were expressed as a specific protein $/ \beta$-actin ratio to minimize the loading variations. Protein bands were visualized by Enhanced Chemiluminescence kit (Beyotime Institute of Biotechnology). The relative chemiluminescent signal intensity was semi-quantified using ImageJ software version 1.48 (National Institutes of Health).

Statistical analysis. All experiments were repeated three times independently. All data are presented as the mean \pm standard error of the mean. Statistical analysis was performed using SPSS software version 23.0 (IBM Corp.). Inter-group variation was assessed by performing one-way analysis of variance and subsequent Tukey's test. $\mathrm{P}<0.05$ was considered to indicate a statistically significant difference.

\section{Results}

Metabolic characterization. The metabolic characteristics of the four groups of BALB/c mice subjected to various treatments are summarized in Fig. 1. The body weight was recorded
$48 \mathrm{~h}$ post-operation did not vary significantly among the four groups. The CLP group exhibited a significant increase in the ALT, AST and ALP levels compared with all other groups; however, these levels were significantly decreased in the TQ + CLP group. There was no significant difference with respect to the aforementioned parameters between the control and TQ groups.

$T Q$ reduces histopathological damage in $L T$. H\&E staining was performed to evaluate the histopathological damage in LT (Fig. 2) and the results for the control and TQ groups revealed that hepatocytes were in regular shape and contained a large spheroidal nucleus. Contrastingly, the CLP group exhibited a marked liver injury demonstrated by hepatic strand disorganization, zonal necrosis, mononuclear cell infiltrations, centrilobular swelling, and sinusoidal and centrilobular congestions compared with the other groups. However, the administration of TQ to mice prevented the degenerative alterations in the hepatic structure induced by CLP.

$T Q$ increases LC3 and beclin 1 expression and decreases $p 62$ expression in $L T$. IHC staining was performed to evaluate the expression levels of LC3 and p62 in LTs (Fig. 3A). The TQ + CLP group exhibited a marked increase in LC3 expression and a decrease in p62 expression in the LT compared with the CLP group. WBA was performed to semi-quantify LC3, beclin 1 and p62 levels (Fig. 3B and C). The present study revealed that the expression levels of LC3 and beclin 1 were significantly increased and the expression levels of p62 were significantly decreased in the TQ + CLP group compared with the CLP group (Fig. 3B and C).

$T Q$ reduces $P I 3 K$ expression in the damaged $L T$. To investigate the effect of TQ on the regulation of the PI3K signaling pathway, the PI3K level was assessed in the treatment groups by performing WBA (Fig. 4A). The present study revealed that PI3K expression was higher in the CLP group compared with the control group. Additionally, the TQ + CLP group exhibited significantly decreased PI3K expression compared with the CLP group (Fig. 4B).

$T Q$ reduces the expression levels of interleukin (IL)-6, IL-1 $\beta$, monocyte chemoattractant protein-1 (MCP-1) and tumor necrosis factor $\alpha(T N F-\alpha)$ but increases IL-10 expression in the LTs of the CLP group. To examine the involvement of inflammatory factors at the gene expression level in the LTs obtained from mice, the expression levels of IL-6, IL-1 $\beta$, MCP-1, IL-10 and TNF- $\alpha$ were quantified using RT-qPCR (Fig. 5). IL-6, IL-1 $\beta$, MCP-1 and TNF- $\alpha$ expression were upregulated in the CLP group; however, this upregulation was attenuated in the TQ + CLP group. By contrast, IL-10 expression was downregulated in the CLP group; however, this was upregulated in the TQ + CLP group.

\section{Discussion}

To the best of our knowledge, TQ affects autophagy in myocardial ischemia-reperfusion (I/R) injury in Langendorffperfused rat hearts and exhibits anti-inflammatory activities in rheumatoid arthritis rat model $(25,26)$. However, limited 

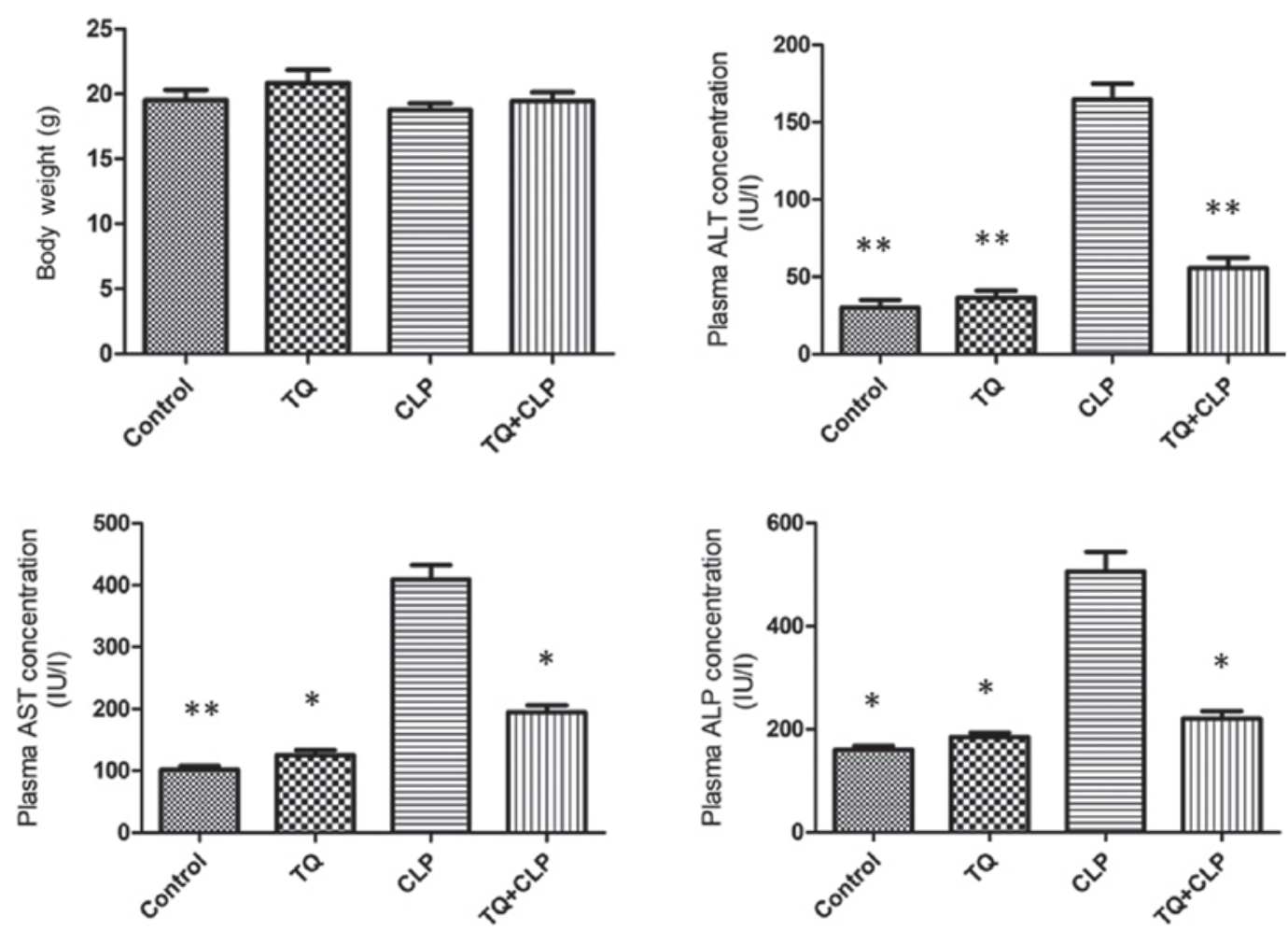

Figure 1. Metabolic data of the four groups of BALB/c mice after various treatments. Body weight, and ALT, AST and ALP levels are presented. Data are represented as the mean $\pm \mathrm{SEM} ; \mathrm{n}=10-12$ per group. ${ }^{*} \mathrm{P}<0.05$ and ${ }^{* * *} \mathrm{P}<0.01$ vs. CLP group. ALP, alkaline phosphatase; ALT, alanine aminotransferase; AST, aspartate aminotransferase; CLP, cecal ligation and puncture; TQ, thymoquinone.
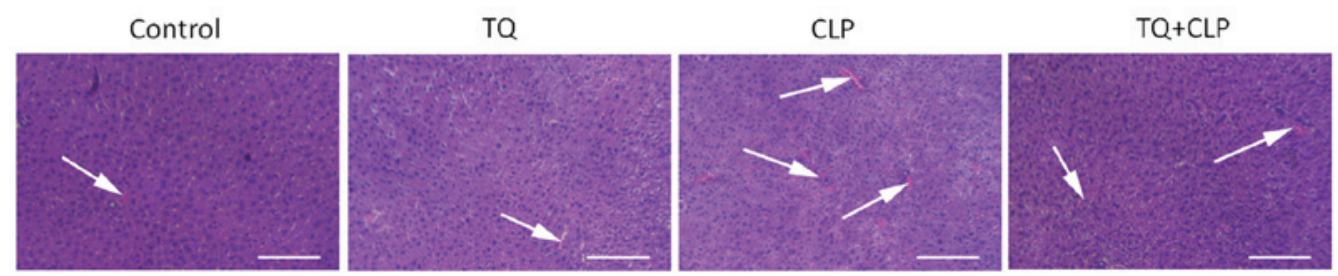

Figure 2. Histopathological damage in the liver tissue in the four groups of BALB/c mice subjected to various treatments. Arrows exhibited hepatic strand disorganization, zonal necrosis, centrilobular swelling, and sinusoidal and centrilobular congestions. Scale bar, $100 \mu \mathrm{m}$. CLP, cecal ligation and puncture; TQ, thymoquinone.

information is available regarding the effect of TQ on CLP-induced septic liver injury. Therefore, the present study investigated the effect of TQ on CLP-induced septic liver injury using a murine model of CLP-induced polymicrobial sepsis. The present study demonstrated that TQ ameliorated septic liver injury as indicated by the regulation of hepatic enzymes (AST, ALT and ALP), autophagy indicators (LC3, p62 and beclin 1), the PI3K signaling pathway and inflammatory factors (IL-6, IL-1 $\beta$, IL-10, MCP- 1 and TNF- $\alpha$ ).

In the present study, no significant variation was observed regarding the increment in body weight among the four groups of mice. The sepsis-induced liver damage could further be investigated by assessment of liver cell integrity markers (AST and ALT) and cholestasis parameters (ALP) (27). The serum levels of hepatic enzymes primarily mirror the degree of liver injury (28) and have been used in the present study as a diagnostic marker for hepatotoxicity. The present study indicated that the serum levels of AST, ALT and ALP were significantly increased in the CLP group compared with in the control group. The TQ group exhibited a prominent reduction in these hepatic biochemical parameters compared with the CLP group. These hepatic biochemical parameters clearly reflected the damage of hepatotoxicity (ALT and AST) and cholestasis (ALP) (27) that was alleviated by TQ. Previous studies indicated that treatment with TQ led to similar results regarding the serum levels of AST, ALT and ALP during lead-induced hepatic toxicity and alcoholic liver injury induced by chronic-plus-binge ethanol feeding in murine models $(29,30)$. Additionally, according to the histological evidence of liver injury, as assessed by performing H\&E staining, treatment of murine models with TQ protected mice against the liver damage caused by CLP-induced sepsis.

Several studies demonstrated a close interaction between CLP-induced sepsis-associated organ failure and cell dysfunction caused by autophagy $(3,31)$. Additionally, a previous study indicated that prominent autophagosomal accumulation was observed in the liver of patients who died of sepsis as well as in the early stages of experimentally-induced sepsis in mouse model (3). In the present study, autophagosome formation was determined by performing IHC analysis and verified using 
A
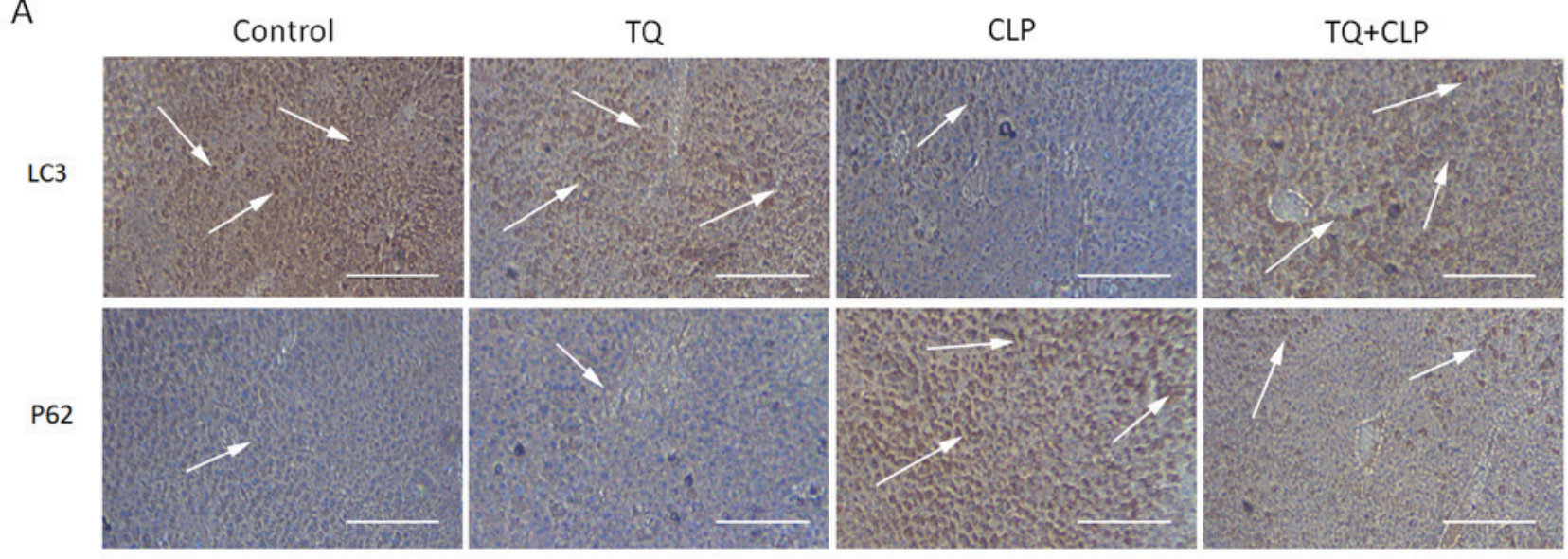

B
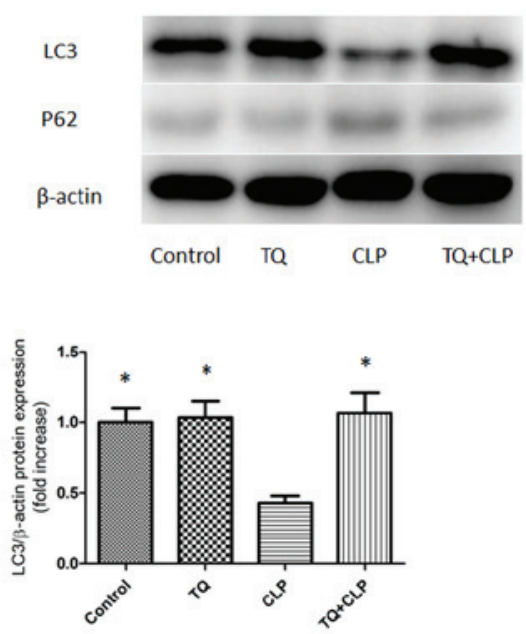
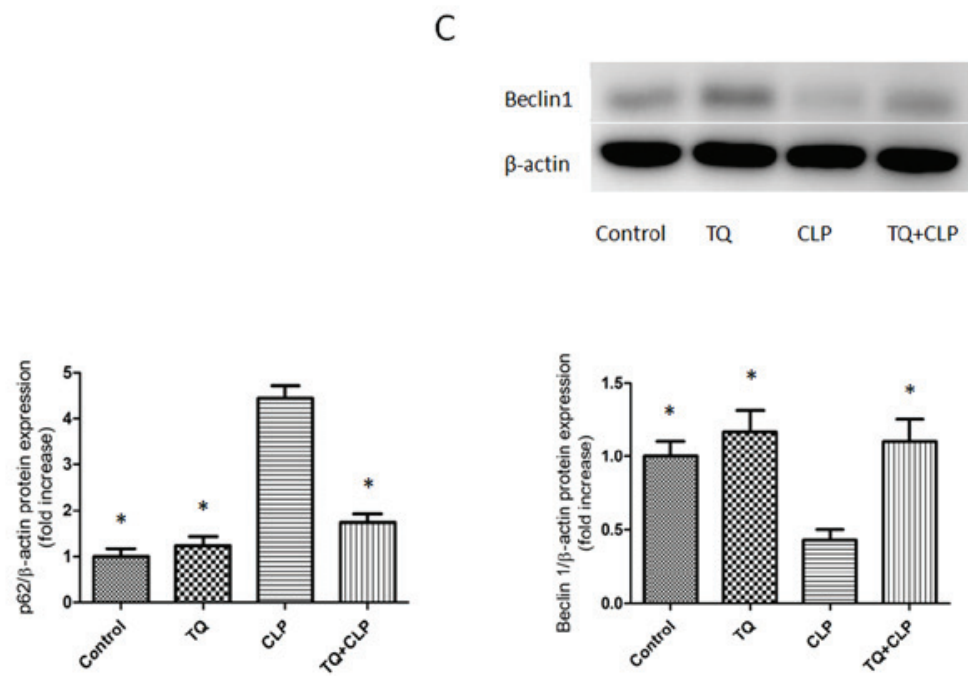

Figure 3. LC3, p62 and beclin 1 expression in LTs of the four groups of BALB/c mice following various treatments. (A) Representative images of immunohistochemical analysis to detect the LC3 and p62 levels in the LTs. Arrows indicate positively stained cells. Scale bar, $100 \mu \mathrm{m}$. Western blot analysis to detect the expression levels of (B) LC3 and p62, and (C) beclin 1 in the LTs. Data are presented as the mean \pm SEM; $n=3-4$ per group. ${ }^{*} \mathrm{P}<0.05$ vs. CLP group. CLP, cecal ligation and puncture; LC3, microtubule-associated protein light chain 3; LT, liver tissue; p62, sequestosome-1; TQ, thymoquinone.
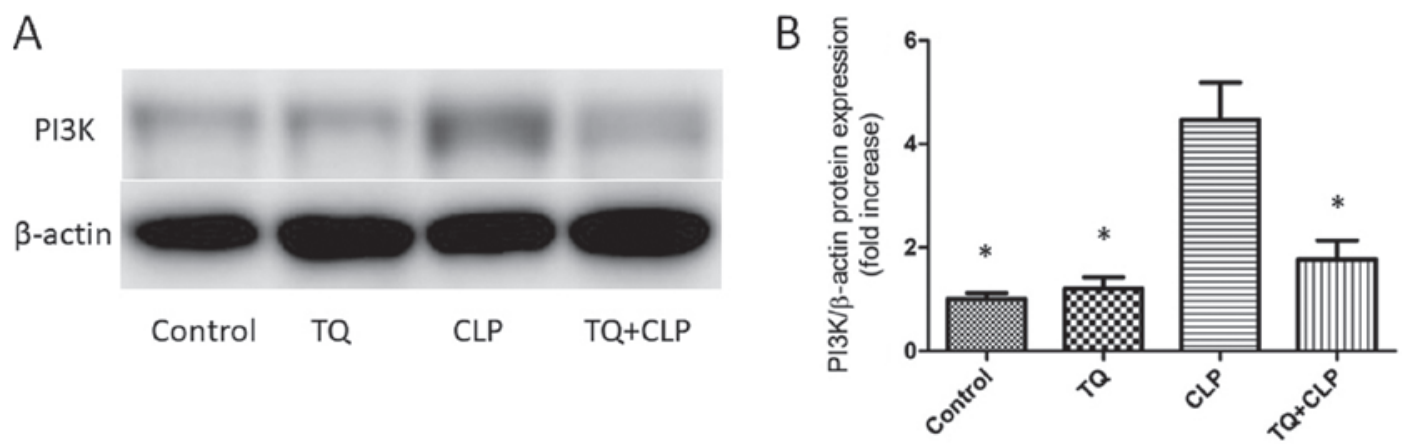

Figure 4. PI3K expression in the LTs of the four groups of BALB/c mice following various treatments. (A) Western blot analysis to detect the PI3K expression in the LTs. (B) Bar graph depicting the semi-quantification of PI3K expression. Data are presented as the mean \pm SEM; $n=3-4$ in each group. " $P<0.05$ vs. CLP group. CLP, cecal ligation and puncture; LT, liver tissue; TQ, thymoquinone.

WBA. The present study revealed that the expression levels of autophagy-specific proteins in the liver were altered in the CLP group compared with in the control group.

LC3, p62 and beclin 1 are three major proteins involved in autophagy (32). The levels of LC3 have been used to assess autophagosome formation (33). The results of the present study indicated that low expression levels of LC3 aggregation in the LT were observed at $48 \mathrm{~h}$ in the CLP group compared with in the control group. Additionally, several studies have indicated a time-course-dependent alteration in LC3 aggregation 

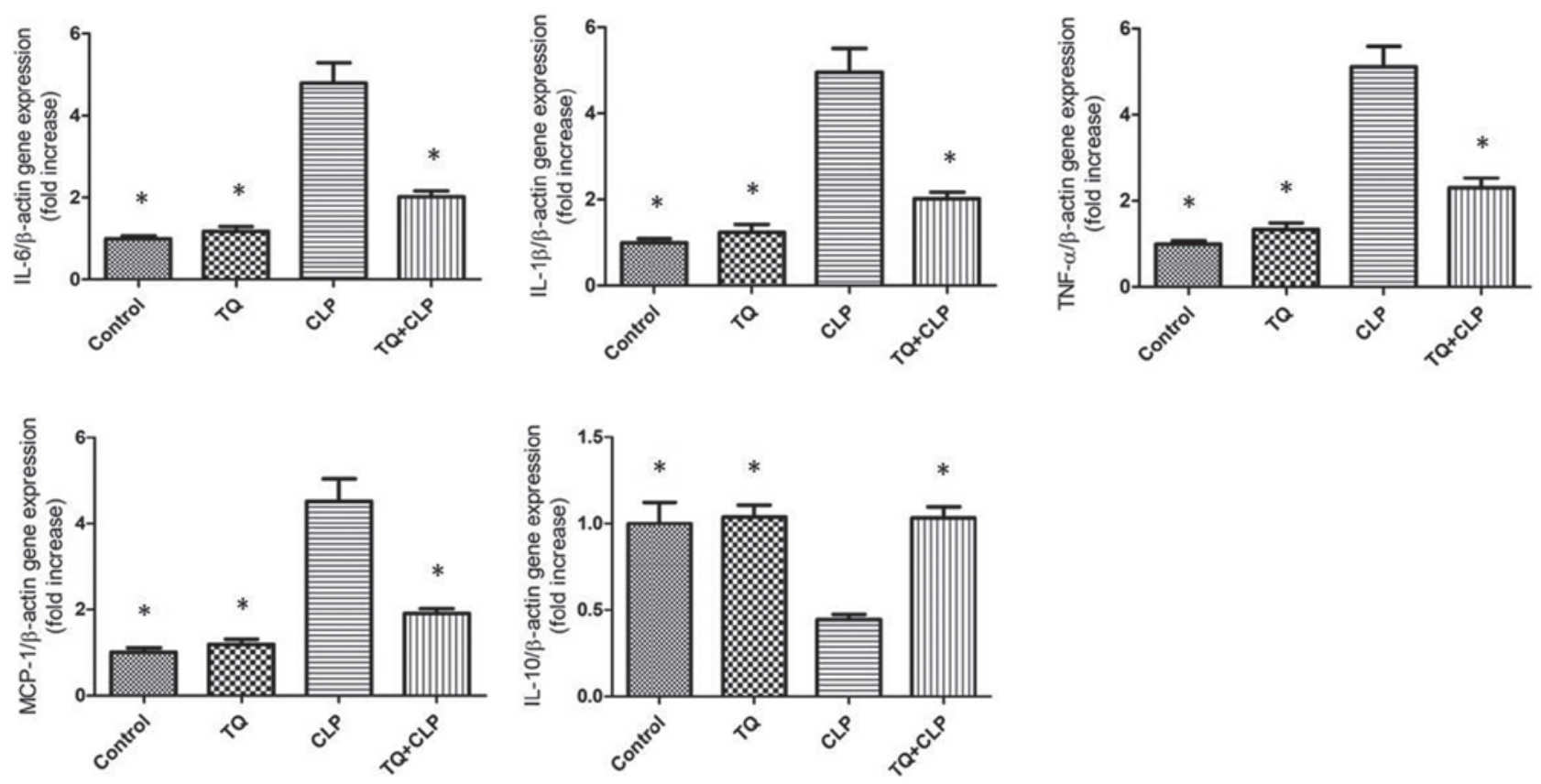

Figure 5. Expression levels of proinflammatory cytokines in the LTs obtained from the four groups of BALB/c mice after various treatments. Relative mRNA expression levels of IL-6, IL-1 $\beta$, MCP-1, IL-10 and TNF- $\alpha$ in the LTs. Data are presented as the means \pm SEM; $n=6$ in each group. "P $<0.05$ vs. CLP group. CLP, cecal ligation and puncture; IL, interleukin; LT, liver tissue; MCP-1, monocyte chemoattractant protein-1; TNF- $\alpha$, tumor necrosis factor $\alpha$; TQ, thymoquinone.

in CLP-induced sepsis. LC3 aggregation was significantly increased at 6-8 $\mathrm{h}$ and gradually decreased $12-24 \mathrm{~h}$ after CLP (34-36). In the present study, LC3 expression in the TQ + CLP group was higher compared with that in the CLP group at $48 \mathrm{~h}$ post-CLP. This indicated that pretreatment with TQ may increase the autophagosome formation in CLP-induced sepsis. As a traditional Chinese medicine that has been used for centuries, multiple studies support the positive effect of TQ in the prevention and cure of various diseases. A previous study reported that TQ dose-dependently increased intracellular LC3 expression in camptothecin-11-resistant LoVo colon cancer cells (37). Additionally, Chu et al (38) reported that TQ treatment increased the accumulation of autophagosomes in human squamous carcinoma cells. As for beclin 1, the present study indicated that pretreatment with TQ may increase expression of beclin 1 in CLP-induced sepsis. Wan et al (36) demonstrated that the expression of autophagy-associated proteins, including beclin 1, declined to basal levels by $12 \mathrm{~h}$ post-CLP. Liu et al (39) revealed that TQ increased beclin 1 gene and protein expression levels; this result was in accordance with the results of the present study. However, high p62 aggregation in the LT was observed in the CLP group compared with in the control group. Pretreatment with TQ after performing CLP reduced the p62 level compared with that in the CLP group. Previous studies have established that p62 is a multidomain adaptor protein that transports ubiquitinated proteins during autophagy. Consistent with the results of the present study, other studies suggest that p62 concentration is generally inversely associated with autophagosomal activity (40-42).

Generally, autophagy is a stringently regulated machinery that degrades and recycles the unnecessary or damaged components of a cell to maintain cellular homeostasis (43). In the present study, TQ was used to prevent CLP-induced septic liver injury in vivo.
In the present study, to investigate the effect of TQ on the regulation of the PI3K signaling pathway, PI3K expression was initially assessed by performing WBA. PI3K represents a key signaling molecule that regulates several cellular functions, including proliferation, survival, adhesion and migration in the liver $(44,45)$. Previous studies suggested that the PI3K pathway is closely associated with autophagy. Li et al (46) reported that several microbial virulent factors induced autophagy by inactivating the PI3K/Akt/mTOR pathway, and Li et al (47) suggested that spironolactone promoted autophagy by inhibiting the PI3K/AKT/mTOR signaling pathway.

A recent study indicated that TQ significantly augmented cisplatin-induced antitumor effects in gastric cancer in vitro as well as in vivo through the inhibition of the PI3K/Akt signaling pathway (48). Furthermore, another study suggested that TQ inhibited PI3K phosphorylation in thioacetamide-induced hepatic fibrosis and inflammation (49). Upon lipopolysaccharide stimulation, the PI3K pathway activation serves to balance between proinflammatory and anti-inflammatory responses (50). Recknagel et al (51) demonstrated that PI3K signaling was spontaneously induced in a rat model with long-term fecal peritonitis. A significantly increased PI3K expression was also observed in the murine liver following aluminum overload (52). In the present study, high PI3K expression was observed in the CLP group compared with in the control group. Conversely, the TQ + CLP group exhibited a reduced PI3K level compared with the CLP group. Collectively, TQ may inhibit PI3K signaling to protect against CLP-induced septic liver injury.

The present study demonstrated that the mRNA expression levels of IL-6, IL-1 $\beta$, MCP-1 and TNF- $\alpha$ in the liver were significantly upregulated in the CLP group compared with those in the control group. In the TQ pretreatment group, the expression levels of IL-6, IL-1 $\beta$, MCP-1 and 
TNF- $\alpha$ in the liver were reduced as a response against CLP. Inversely, IL-10 expression was downregulated in the CLP group; however, its expression levels were upregulated in the TQ + CLP group. Experimental studies indicated that TQ reduced the production of IL- 6 , IL-1 $\beta$, MCP-1 and TNF- $\alpha$ in the blood and other tissues (53). Furthermore, a previous study reported that oral administration of TQ results in significantly increased expression levels of IL-10 in the collagen-induced arthritis in Wistar rats model (54). Additionally, Hsiao et al (55) reported that treatment using an inducer of autophagy diminished TNF- $\alpha$-induced DNA fragmentation. Additionally, Giegerich et al (56) illustrated that autophagy-dependent pellino E3 ubiquitin protein ligase family member 3 degradation inhibited proinflammatory IL-1 $\beta$ expression.

In conclusion, the present study demonstrated that TQ assisted the mitigation of septic liver injury, as indicated by the upregulation of LC3, beclin 1 and IL-10 expression as well as the suppression of p62, PI3K, IL-6, IL-1 $\beta$, MCP-1 and TNF- $\alpha$ expression. These findings provided novel insights into the role of TQ in sepsis-induced liver injury, and improve the possibility of developing novel therapeutic interventions to treat liver injury.

\section{Acknowledgements}

Not applicable.

\section{Funding}

The present study was finally supported by the Postdoctoral Foundation of Liaoning Province, China (grant no. 194008).

\section{Availability of data and materials}

All data generated or analyzed during this study are included in this published article.

\section{Authors' contributions}

ZP designed this study. FW and XL performed experiments. ZP, HZ, YZ and QY analyzed the data, interpreted the results of the experiments and prepared the figures. QL analyzed the data, interpreted the results of the experiments and prepared the figures. XL drafted the manuscript. ZP, QL and YZ contributed to the revision of the manuscript. All authors read and approved the final manuscript.

\section{Ethics approval and consent to participate}

All animal experiments were approved by the Animal Studies Committee of the Affiliated Zhongshan Hospital of Dalian University.

\section{Patient consent for publication}

Not applicable.

\section{Competing interests}

The authors declare that they have no competing interests.

\section{References}

1. Singer M, Deutschman CS, Seymour CW, Shankar-Hari M, Annane D, Bauer M, Bellomo R, Bernard GR, Chiche JD, Coopersmith CM, et al: The third international consensus definitions for sepsis and septic shock (Sepsis-3). JAMA 315: 801-810, 2016.

2. Alkharfy KM, Ahmad A, Jan BL and Raish M: Thymoquinone reduces mortality and suppresses early acute inflammatory markers of sepsis in a mouse model. Biomed Pharmacother 98: 801-805, 2018 .

3. Lin CW, Lo S, Perng DS, Wu DB, Lee PH, Chang YF, Kuo PL, Yu ML, Yuan SS and Hsieh YC: Complete activation of autophagic process attenuates liver injury and improves survival in septic mice. Shock 41: 241-249, 2014.

4. Xiong X, Ren Y, Cui Y, Li R, Wang C and Zhang Y: Obeticholic acid protects mice against lipopolysaccharide-induced liver injury and inflammation. Biomed Pharmacother 96: 1292-1298, 2017.

5. Marshall JC: Why have clinical trials in sepsis failed? Trends Mol Med 20: 195-203, 2014.

6. Lalazar G, Ilyas G, Malik SA, Liu K, Zhao E, Amir M, Lin Y, Tanaka KE and Czaja MJ: Autophagy confers resistance to lipopolysaccharide-induced mouse hepatocyte injury. Am J Physiol Gastrointest Liver Physiol 311: G377-G386, 2016.

7. Hsieh CH, Pai PY, Hsueh HW, Yuan SS and Hsieh YC: Complete induction of autophagy is essential for cardioprotection in sepsis. Ann Surg 253: 1190-1200, 2011.

8. Zhang J, Zhao P, Quan N, Wang L, Chen X, Cates C, Rousselle T and $\mathrm{Li} \mathrm{J}$ : The endotoxemia cardiac dysfunction is attenuated by AMPK/mTOR signaling pathway regulating autophagy. Biochem Biophys Res Commun 492: 520-527, 2017.

9. Li T, Zhao J, Miao S, Xu Y, Xiao X and Liu Y: Dynamic expression and roles of sequestome1/p62 in LPSinduced acute kidney injury in mice. Mol Med Rep 17: 7618-7626, 2018.

10. Watanabe E, Muenzer JT, Hawkins WG, Davis CG, Dixon DJ, McDunn JE, Brackett DJ, Lerner MR, Swanson PE and Hotchkiss RS: Sepsis induces extensive autophagic vacuolization in hepatocytes: A clinical and laboratory-based study. Lab Invest 89: 549-561, 2009.

11. Lo S, Yuan SS, Hsu C, Cheng YJ, Chang YF, Hsueh HW, Lee PH and Hsieh YC: Lc3 over-expression improves survival and attenuates lung injury through increasing autophagosomal clearance in septic mice. Ann Surg 257: 352-363, 2013.

12. Ni HM, Jaeschke H and Ding WX: Targeting autophagy for drug-induced hepatotoxicity. Autophagy 8: 709-710, 2012.

13. Rautou PE, Mansouri A, Lebrec D, Durand F, Valla D and Moreau R: Autophagy in liver diseases. J Hepatol 53: 1123-1134, 2010.

14. Oami T, Watanabe E, Hatano M, Teratake Y, Fujimura L, Sakamoto A, Ito C, Toshimori K, Swanson PE and Oda S: Blocking liver autophagy accelerates apoptosis and mitochondrial injury in hepatocytes and reduces time to mortality in a murine sepsis model. Shock 50: 427-434, 2018.

15. Oami T, Watanabe E, Hatano M, Sunahara S, Fujimura L, Sakamoto A, Ito C, Toshimori K and Oda S: Suppression of t cell autophagy results in decreased viability and function of $\mathrm{t}$ cells through accelerated apoptosis in a murine sepsis model. Crit Care Med 45: e77-e85, 2017.

16. Daba MH and Abdel-Rahman MS: Hepatoprotective activity of thymoquinone in isolated rat hepatocytes. Toxicol Lett 95: 23-29, 1998.

17. Khan MA, Ashfaq MK, Zuberi HS, Mahmood MS and Gilani AH: The in vivo antifungal activity of the aqueous extract from Nigella sativa seeds. Phytother Res 17: 183-186, 2003.

18. Houghton PJ, Zarka R, de las Heras B and Hoult JR: Fixed oil of Nigella sativa and derived thymoquinone inhibit eicosanoid generation in leukocytes and membrane lipid peroxidation. Planta Med 61: 33-36, 1995.

19. Reddy L, Odhav B and Bhoola KD: Natural products for cancer prevention: A global perspective. Pharmacol Ther 99: 1-13, 2003.

20. Salem ML: Immunomodulatory and therapeutic properties of the Nigella sativa L. seed. Int Immunopharmacol 5: 1749-1770, 2005. 
21. Darakhshan S, Bidmeshki Pour A, Hosseinzadeh Colagar A and Sisakhtnezhad S: Thymoquinone and its therapeutic potentials. Pharmacol Res 95-96: 138-158, 2015.

22. Rittirsch D, Huber-Lang MS, Flierl MA and Ward PA: Immunodesign of experimental sepsis by cecal ligation and puncture. Nat Protoc 4: 31-36, 2009.

23. National Research Council (US) Committee for the Update of the Guide for the Care and Use of Laboratory Animals: Guide for the Care and Use of Laboratory Animals. 8th edition. National Academies Press (US), Washington, D.C., 2011.

24. Livak KJ and Schmittgen TD: Analysis of relative gene expression data using real-time quantitative PCR and the 2(-Delta Delta C(T)) method. Methods 25: 402-408, 2001.

25. Tekeoglu I, Dogan A and Demiralp L: Effects of thymoquinone (volatile oil of black cumin) on rheumatoid arthritis in rat models. Phytother Res 20: 869-871, 2006.

26. Xiao J, Ke ZP, Shi Y, Zeng Q and Cao Z: The cardioprotective effect of thymoquinone on ischemia-reperfusion injury in isolated rat heart via regulation of apoptosis and autophagy. J Cell Biochem 119: 7212-7217, 2018.

27. Penndorf V, Saner F, Gerken G and Canbay A: Liver parameters in intensive care medicine. Zentralbl Chir 138: 636-642, 2013 (In German).

28. Hsu DZ, Chien SP, Li YH and Liu MY: Sesame oil does not show accumulatively enhanced protection against oxidative stress-associated hepatic injury in septic rats. JPEN J Parenter Enteral Nutr 32: 276-280, 2008.

29. Mabrouk A, Bel Hadj Salah I, Chaieb W and Ben Cheikh H: Protective effect of thymoquinone against lead-induced hepatic toxicity in rats. Environ Sci Pollut Res Int 23: 12206-12215, 2016

30. Yang Y, Bai T, Yao YL, Zhang DQ, Wu YL, Lian LH and Nan JX: Upregulation of SIRT1-AMPK by thymoquinone in hepatic stellate cells ameliorates liver injury. Toxicol Lett 262: 80-91, 2016.

31. Carchman EH, Whelan S, Loughran P, Mollen K, Stratamirovic S, Shiva S, Rosengart MR and Zuckerbraun BS: Experimental sepsis-induced mitochondrial biogenesis is dependent on autophagy, TLR4, and TLR9 signaling in liver. FASEB J 27: 4703-4711, 2013.

32. Schmitz KJ, Ademi C, Bertram S, Schmid KW and Baba HA: Prognostic relevance of autophagy-related markers LC3, p62/sequestosome 1, Beclin-1 and ULK1 in colorectal cancer patients with respect to KRAS mutational status. World J Surg Oncol 14: 189, 2016.

33. Escobar DA, Botero-Quintero AM, Kautza BC, Luciano J, Loughran P, Darwiche S, Rosengart MR, Zuckerbraun BS and Gomez H: Adenosine monophosphate-activated protein kinase activation protects against sepsis-induced organ injury and inflammation. J Surg Res 194: 262-272, 2015.

34. Takahashi W, Watanabe E, Fujimura L, Watanabe-Takano $\mathrm{H}$, Yoshidome H, Swanson PE, Tokuhisa T, Oda S and Hatano M: Kinetics and protective role of autophagy in a mouse cecal ligation and puncture-induced sepsis. Crit Care 17: R160, 2013.

35. Sunahara S, Watanabe E, Hatano M, Swanson PE, Oami T, Fujimura L, Teratake Y, Shimazui T, Lee C and Oda S: Influence of autophagy on acute kidney injury in a murine cecal ligation and puncture sepsis model. Sci Rep 8: 1050, 2018.

36. Wan SX, Shi B, Lou XL, Liu JQ, Ma GG, Liang DY and Ma S: Ghrelin protects small intestinal epithelium against sepsis-induced injury by enhancing the autophagy of intestinal epithelial cells. Biomed Pharmacother 83: 1315-1320, 2016.

37. Chen MC, Lee NH, Hsu HH, Ho TJ, Tu CC, Hsieh DJ, Lin YM, Chen LM, Kuo WW and Huang CY: Thymoquinone induces caspase-independent, autophagic cell death in CPT-11-resistant lovo colon cancer via mitochondrial dysfunction and activation of JNK and p38. J Agric Food Chem 63: 1540-1546, 2015.

38. Chu SC, Hsieh YS, Yu CC, Lai YY and Chen PN: Thymoquinone induces cell death in human squamous carcinoma cells via caspase activation-dependent apoptosis and LC3-II activation-dependent autophagy. PLoS One 9: e101579, 2014.

39. Liu H, Sun Y, Zhang Y, Yang G, Guo L, Zhao Y and Pei Z: Role of thymoquinone in cardiac damage caused by sepsis from $\mathrm{BALB} / \mathrm{c}$ mice. Inflammation 42: 516-525, 2019.

40. Zhao P, Kuai J, Gao J, Sun L, Wang Y and Yao L: Delta opioid receptor agonist attenuates lipopolysaccharide-induced myocardial injury by regulating autophagy. Biochem Biophys Res Commun 492: 140-146, 2017.
41. Wang GQ, Tang T, Wang ZS, Liu YY, Wang L, Luo PF and Xia ZF: Overexpression of hypo-phosphorylated I $\kappa B \beta$ at ser313 protects the heart against sepsis. PLoS One 11: e0160860, 2016

42. Wang H, Cui N, Han W, Su LX, Long Y and Liu DW: Accelerated autophagy of cecal ligation and puncture-induced myocardial dysfunction and its correlation with mammalian target of rapamycin pathway in rats. Chin Med J (Engl) 131: 1185-1190, 2018.

43. Chung KW, Kim KM, Choi YJ, An HJ, Lee B, Kim DH, Lee EK, Im E, Lee J, Im DS, et al: The critical role played by endotoxin-induced liver autophagy in the maintenance of lipid metabolism during sepsis. Autophagy 13: 1113-1129, 2017.

44. Miao B and Degterev A: Targeting phospshatidylinositol 3-kinase signaling with novel phosphatidylinositol 3,4,5-triphosphate antagonists. Autophagy 7: 650-651, 2011.

45. Reif S, Lang A, Lindquist JN, Yata Y, Gabele E, Scanga A, Brenner DA and Rippe RA: The role of focal adhesion kinase-phosphatidylinositol 3-kinase-akt signaling in hepatic stellate cell proliferation and type I collagen expression. J Biol Chem 278: 8083-8090, 2003.

46. Li P, Shi J, He Q, Hu Q, Wang YY, Zhang LJ, Chan WT and Chen WX: Streptococcus pneumoniae induces autophagy through the inhibition of the PI3K-I/Akt/mTOR pathway and ROS hypergeneration in A549 cells. PLoS One 10: e0122753, 2015.

47. Li D, Lu Z, Xu Z, Ji J, Zheng Z, Lin S and Yan T: Spironolactone promotes autophagy via inhibiting PI3K/AKT/mTOR signalling pathway and reduce adhesive capacity damage in podocytes under mechanical stress. Biosci Rep 36: e00355, 2016.

48. Ma J, Hu X, Li J, Wu D, Lan Q, Wang Q, Tian S and Dong W: Enhancing conventional chemotherapy drug cisplatin-induced anti-tumor effects on human gastric cancer cells both in vitro and in vivo by thymoquinone targeting PTEN gene. Oncotarget 8 : 85926-85939, 2017.

49. Bai T, Yang Y, Wu YL, Jiang S, Lee JJ, Lian LH and Nan JX: Thymoquinone alleviates thioacetamide-induced hepatic fibrosis and inflammation by activating LKB1-AMPK signaling pathway in mice. Int Immunopharmacol 19: 351-357, 2014.

50. Yang JC, Wu SC, Rau CS, Lu TH, Wu YC, Chen YC, Lin MW, Tzeng SL, Wu CJ and Hsieh CH: Inhibition of the phosphoinositide 3-kinase pathway decreases innate resistance to lipopolysaccharide toxicity in TLR4 deficient mice. J Biomed Sci 21: 20, 2014.

51. Recknagel P, Gonnert FA, Westermann M, Lambeck S, Lupp A, Rudiger A, Dyson A, Carre JE, Kortgen A, Krafft C, et al: Liver dysfunction and phosphatidylinositol-3-kinase signalling in early sepsis: Experimental studies in rodent models of peritonitis. PLoS Med 9: e1001338, 2012

52. Hu C, Yang J, He Q, Luo Y, Chen Z, Yang L, Yi H, Li H, Xia H, Ran D, et al: CysLTR1 blockage ameliorates liver injury caused by aluminum-overload via PI3K/AKT/mTOR-mediated autophagy activation in vivo and in vitro. Mol Pharm 15: 1996-2006, 2018.

53. Ghazwani M, Zhang Y, Gao X, Fan J, Li J and Li S: Anti-fibrotic effect of thymoquinone on hepatic stellate cells. Phytomedicine 21: 254-260, 2014.

54. Umar S, Zargan J, Umar K, Ahmad S, Katiyar CK and Khan HA: Modulation of the oxidative stress and inflammatory cytokine response by thymoquinone in the collagen induced arthritis in Wistar rats. Chem Biol Interact 197: 40-46, 2012

55. Hsiao HW, Tsai KL, Wang LF, Chen YH, Chiang PC, Chuang SM and Hsu C: The decline of autophagy contributes to proximal tubular dysfunction during sepsis. Shock 37: 289-296, 2012

56. Giegerich AK, Kuchler L, Sha LK, Knape T, Heide H, Wittig I, Behrends C, Brüne B and von Knethen A: Autophagy-dependent PELI3 degradation inhibits proinflammatory IL1B expression. Autophagy 10: 1937-1952, 2014.

This work is licensed under a Creative Commons Attribution-NonCommercial-NoDerivatives 4.0 International (CC BY-NC-ND 4.0) License. 